\title{
Americans' COVID-19 Stress, Coping, and Adherence to CDC Guidelines
}

\author{
Crystal L. Park, Ph.D. ${ }^{\top}\left(0\right.$, Beth S. Russell, Ph.D. ${ }^{2}$, Michael Fendrich, Ph.D. ${ }^{3}$, \\ Lucy Finkelstein-Fox, MS ${ }^{7}$, Morica Hutchison, $\mathrm{MFT}^{2}$, and Jessica Becker, MSW ${ }^{3}$
}

'Department of Psychological Sciences, University of Connecticut, Mansfield, CT, USA; ${ }^{2}$ Department of Human Development and Family Studies, University of Connecticut, Storrs, CT, USA; ${ }^{3}$ School of Social Work, University of Connecticut, 38 Prospect Street, Harford, CT, USA.

IMPORTANCE: Documenting Americans' stress responses to an unprecedented pandemic and their degree of adherence to CDC guidelines is essential for mental health interventions and policy-making.

OBJECTIVE: To provide the first snapshot of immediate impact of COVID-19 on Americans' stress, coping, and guideline adherence.

DESIGN: Data were collected from an online workers' platform for survey research (Amazon's Mechanical Turk) from April 7 to 9, 2020. The current data represents the baseline of a longitudinal study. Best practices for ensuring high-quality data were employed.

PARTICIPANTS: Individuals who are 18 years of age or older, living in the USA, and English-speaking were eligible for the study. Of 1086 unique responses, 1015 completed responses are included.

SETTING: Population-based.

MAIN OUTCOMES: Exposure to and stressfulness of COVID-19 stressors, coping strategies, and adherence to CDC guidelines.

RESULTS: The sample was 53.9\% women ( $n=547)$, with an average age of 38.9 years $(\mathrm{SD}=13.50$, range $=18-88)$, most of whom were White ( $n=836,82.4 \%)$, non-Hispanic ( $n=929,91.5 \%)$, and straight/heterosexual $(n=895$, $88.2 \%) ; 40 \%$ were currently married $(n=407)$, and $21.6 \%(n=219)$ were caregivers. About half $(50.5 \%)$ endorsed having at least "mostly" enough money to meet their needs. Respondents' locations across the USA ranged from $18.5 \%$ in the Northeast to $37.8 \%$ in the South. The most commonly experienced stressors were reading/hearing about the severity and contagiousness of COVID-19, uncertainty about length of quarantine and social distancing requirements, and changes to social and daily personal care routines. Financial concerns were rated most stressful. Younger age, female gender, and caregiver status increased risk for stressor exposure and greater degree of stressfulness. The most frequently

Key Points Question: How are Americans responding to COVID-19 in terms of stressors, coping, and adherence?

Findings: Americans have high COVID-19 stress exposure and some demographic subgroups appear particularly vulnerable to stress effects. Subgroups less likely to adhere to CDC guidelines (e.g., men, younger adults) may benefit from targeted information campaigns.

Meaning: The documented high levels of stress and suboptimal adherence to $C D C$ guidelines may guide public health and mental health interventions and inform policu-makina.

Received April 17, 2020

Accepted April 30, 2020

Published online May 29, 2020 reported strategies to manage stress were distraction, active coping, and seeking emotional social support. CDC guideline adherence was generally high, but several key social distancing and hygiene behaviors showed suboptimal adherence, particularly for men and younger adults.

CONCLUSIONS AND RELEVANCE: Americans have high COVID-19 stress exposure and some demographic subgroups appear particularly vulnerable to stress effects. Subgroups less likely to adhere to CDC guidelines may benefit from targeted information campaigns. these findings may guide mental health interventions and inform policy-making regarding implications of specific public health measures.

J Gen Intern Med 35(8):2296-303

DOI: $10.1007 / \mathrm{s} 11606-020-05898-9$

(C) Society of General Internal Medicine 2020

$\mathrm{T}$ he rapid emergence of the COVID-19 pandemic in the USA and subsequent state and federal prevention measures now in place have dramatically altered daily behavior, with a substantial impact on the physical, mental, social, and financial well-being of the population. Nationwide, individuals must navigate school closures, employment insecurity, and changes in social behavior that likely have major adverse effects on their mental health and ability to cope. Indeed, the Centers for Disease Control $^{1}$ has emphasized the need for all individuals to manage stress and protect their mental health during this extremely uncertain time, recognizing that increased stress may lead to maladaptive behaviors to cope with stress and anxiety.

Time-sensitive research has identified diverse stressors in previous geographically limited quarantines, including fears of infection, disruptions to work/learning, and daily self-care routines, in addition to lack of access to reliable information and resources. ${ }^{2}$ However, little is known about these stressors in the midst of an ongoing, open-ended, universally experienced pandemic. Several studies in China suggested only modest impacts on stress during the early months of the pandemic in late 2019 and early 2020. ${ }^{3,4}$ For example, less than one-third of one national sample in China reported elevated stress. $^{5}$ 
Perhaps compounding the stress of COVID-19, the CDC and many government agencies have issued guidelines regarding social distancing and hygiene. Yet during crises, seeking social support is often one of the most adaptive ways to cope with stress. ${ }^{6}$ Thus, many of the very recommendations provided by the CDC to prevent the spread of COVID- $19^{7}$ may reduce critical social supports necessary for well-being. ${ }^{6,8}$ Many other strategies for coping with stress, such as active coping to alleviate problems, have been shown to be helpful during a crisis; others, such as substance use coping, have been found universally maladaptive, and for still others, such as distraction, their utility may depend on the context.9, 10

Very little information is available regarding adherence to COVID-related CDC guidelines to prevent the spread of this disease. One Chinese study reported varying levels of adherence to five recommended behaviors: always wash the hands after touching contaminated objects $(66.6 \%)$, always wear a mask regardless of the presence or absence of symptoms $(59.8 \%)$, always cover the mouth when coughing and sneezing (57.4\%); always wash the hands with soap (56.5\%), always wash the hands immediately after coughing, sneezing, or rubbing the nose $(41 \%)$, and always avoid sharing utensils during meals (40.5\%). ${ }^{5}$ In that study, social distancing adherence was not assessed.

This paper presents the first national US survey results of Americans' experiences in social isolation in early April 2020, including the specific stressors they encountered, the types of coping they employed to manage these stressors, and the extent to which they adhered to current CDC guidelines. Further, exploratory analyses describe how demographic characteristics relate to people's responses to the pandemic. Such information may be critical to interventionists seeking to alleviate adverse mental health effects of isolation as well as to policy-makers in weighing the implications of specific public health measures.

\section{METHODS}

\section{Data Collection}

All study materials were approved by the University of Connecticut IRB (X20-0057) as an exempt protocol. Participants from Amazon's Mechanical Turk (MTurk) online worker pool gave consent prior to completing baseline questionnaires on the impact of COVID-19 on daily life using an anonymous, longitudinal design based on best practices. ${ }^{11}$ At each timepoint, participants receive a $\$ 2$ incentive. Data presented here include results from the baseline survey collected between April 7 and 9, 2020, approximately 3 weeks after quarantines began rolling out across the USA.

\section{Sample}

Individuals who are 18 years of age or older, live in the USA, and are English-speaking were eligible for the study. We received 1086 unique responses to the baseline study online using
MTurk. After removing duplicates and incomplete or substandard responses, the final baseline sample reported here includes 1015 cases (see the "Analysis" section for details). MTurk workers are more diverse than typical student or online forum samples and fairly representative of larger populations, including the USA. ${ }^{12-14}$ Evaluations of the reliability and validity of MTurk have found data to be high-quality, replicable, and valid across comparisons with frequently used academic platforms and student and professional samples. ${ }^{12,15,16}$ Underscoring the utility of this platform for health research, a review of 35 health and medical studies that compared MTurk responses with responses from data obtained from other sources concluded that the MTurk responses were "largely comparable."

\section{Key Measures}

Demographics. Participants reported on their location by state, student enrollment/employment status, financial security ("Do you have enough money to meet your needs," rated from 1 ("not at all") to 5 ("completely"), whether they were a primary caregiver for a dependent, partner status, gender, sexual orientation, race, ethnicity, and age. For descriptive purposes, states were categorized into four distinct regions of the USA based on divisions used in the US Census ${ }^{18}$ (West, Midwest, Northeast, and South).

COVID-19 Stressors. Based on previous work during SARS and the early months of the COVID-19 pandemic, we created an assessment of COVID-19 stressors (based on ${ }^{2,3,19}$ ). This novel measure assesses whether individuals experienced each of 23 stressors in the past week, and for those experienced, ratings of the degree of stressfulness of each from 1 ("not at all stressful") to 5 ("extremely stressful").

CDC Guideline Adherence. Participants rated their degree of adherence (from 0 to $100 \%$ of the time) over the past week with each of 15 items regarding social distancing behavior, health behavior, and hygiene recommendations based on public guidance to prevent the spread of COVID-19 issued by the CDC in March of $2020 .^{7}$

Coping. The survey included the COPE substance use/active coping/self-distraction/behavioral disengagement/humor (18 items) ${ }^{20}$ which asked about how individuals had been coping with "COVID-19-related stressors" over the past week on a 4point Likert scale ranging from 1 ("I haven't been doing this at all") to 4 ("I've been doing this a lot").

\section{Analysis}

Univariate analyses (mean/median, standard deviation, and percentages) and bivariate analyses (chi-square, independent samples $t$ test, bivariate correlation) are reported. 
A priori power analysis with a two-tailed alpha set to .05 suggested that a sample size of $N=1050$ would provide $80 \%$ power to detect small $(d=0.20)$ group differences in groups imbalanced at a 3:1 ratio and approximately $90 \%$ power to detect small $(d=0.20)$ group differences in groups equally balanced at a 1:1 ratio (the same logic applies to $h$ 's, effect sizes for categorical comparisons). Best practices for withholding payment and removal of participants from a dataset include subjective inattentiveness, such as abnormally quick response times, psychometric antonyms, or those deemed as substandard work. ${ }^{16}$, 21,22 A close examination of the raw dataset revealed twelve sets of completed duplicate responses and 59 responses completed in under $10 \mathrm{~min}$ (less than half of the expected time for the survey, based on pilot testing). Duplicate responses were deleted, retaining the first completed response; responses completed in less than $10 \mathrm{~min}$ were also excluded from the analyses presented below to ensure high response quality. The final dataset consisted of 1015 unique participants. Likely due to MTurk's policy that participants submitting incomplete data may not receive incentive, all included participants completed $100 \%$ of the survey; missingness for all items was $<1 \%$. Due to these extremely low rates of missing data, list-wise deletion was used for analyses below. Data cleaning and primary study analyses were conducted in SPSS (version 26); standardized effect sizes were calculated in $\mathrm{R}$ (version 3.6.2). For parsimony, only significant standardized effect sizes are reported in the text below; given the considerable statistical power afforded by this large sample, effect sizes for statistically non-significant group differences are very small to negligible (e.g., $d$ or $h<0.20$ ).

\section{RESULTS}

Our sample was fairly equal in gender $(n=547,53.9 \%$ females), with an average age of 38.9 years old (SD = 13.5, range $=18-88)$ and most participants identifying as White $(n=836,82.4 \%)$, non-Hispanic $(n=929,91.5 \%)$, and straight/heterosexual $(n=895,88.2 \%) ; 40 \%$ were currently married $(n=407)$, and $21.6 \%$ (219) reported being a caregiver. About half $(50.5 \%)$ of participants endorsed having at least "mostly" enough money to meet their needs. Participants reported current locations across the USA (ranging from $18.5 \%$ in the Northeast to $37.8 \%$ in the South). Sixty-two participants $(6.1 \%)$ reported feeling ill, having a fever, or testing positive for COVID-19 during the past week. Demographics are reported in greater detail in Table 1.

\section{COVID-19 STRESSORS}

Stressor exposure was variable but overall, quite high (see Table 2). The most commonly experienced stressors were
Table 1 Demographic Characteristics of the Sample

\begin{tabular}{|c|c|}
\hline \multicolumn{2}{|l|}{ Demographic } \\
\hline Age (years), $M(S D)$ & $38.9(13.5)$ \\
\hline \multicolumn{2}{|l|}{ Gender, $N(\%)$} \\
\hline Male & $453(44.6 \%)$ \\
\hline Female & $547(53.9 \%)$ \\
\hline Non-binary/third gender & $3(0.3 \%)$ \\
\hline Transgender & $4(0.4 \%)$ \\
\hline Prefer to self-describe & $4(0.4 \%)$ \\
\hline Prefer not to say & $4(0.4 \%)$ \\
\hline \multicolumn{2}{|l|}{ Race, $N(\%)$} \\
\hline Black/African American & $122(12.0 \%)$ \\
\hline Asian/Asian American & $121(11.9 \%)$ \\
\hline Native Hawaiian/other Pacific Islander & $50(4.9 \%)$ \\
\hline American Indian/Alaska Native & $70(6.9 \%)$ \\
\hline White & $836(82.4 \%)$ \\
\hline \multicolumn{2}{|l|}{ Ethnicity, $N(\%)$} \\
\hline Hispanic & $86(8.5 \%)$ \\
\hline \multirow{2}{*}{\multicolumn{2}{|c|}{ Sexual orientation, $N(\%)$}} \\
\hline & \\
\hline Straight/heterosexual & $895(88.2 \%)$ \\
\hline Gay or lesbian & $40(3.9 \%)$ \\
\hline Bisexual & $64(6.3 \%)$ \\
\hline Prefer to self-describe & $7(0.7 \%)$ \\
\hline Prefer not to say & $9(0.9 \%)$ \\
\hline \multicolumn{2}{|l|}{ Geographic state, $N(\%)$} \\
\hline West & $244(24.1 \%)$ \\
\hline Midwest & $199(19.6 \%)$ \\
\hline South & $383(37.7 \%)$ \\
\hline Northeast & $188(18.5 \%)$ \\
\hline \multicolumn{2}{|l|}{ Marital status, $N(\%)$} \\
\hline Married & $407(40.1 \%)$ \\
\hline Single & $359(35.4 \%)$ \\
\hline Divorced & $73(7.2 \%)$ \\
\hline Separated & $14(1.4 \%)$ \\
\hline Widowed & $18(1.8 \%)$ \\
\hline Living with but not married & $144(14.2 \%)$ \\
\hline \multicolumn{2}{|l|}{ Current living arrangement, $N(\%)$} \\
\hline In parent/guardian home & $158(15.6 \%)$ \\
\hline With others (no relation) & $214(21.1 \%)$ \\
\hline With others (related) & $468(46.1 \%)$ \\
\hline By yourself & $172(16.9 \%)$ \\
\hline \multicolumn{2}{|l|}{ Residence location, $N(\%)$} \\
\hline House & $614(60.5 \%)$ \\
\hline Apartment & $243(23.9 \%)$ \\
\hline \multicolumn{2}{|l|}{ Caregiver status, $N(\%)$} \\
\hline Yes & $219(21.6 \%)$ \\
\hline No & $796(78.4 \%)$ \\
\hline \multicolumn{2}{|l|}{ Type of caregiver, $N(\%)$} \\
\hline Child (under 18 years old) & $182(83.1 \%)$ \\
\hline Same age relative/friend & $12(5.5 \%)$ \\
\hline Parent & $19(8.7 \%)$ \\
\hline Grandparent & $4(1.8 \%)$ \\
\hline Other & $2(0.9 \%)$ \\
\hline Current student status, $N(\%)$ & \\
\hline No & $890(87.7 \%)$ \\
\hline Yes, part-time & $60(5.9 \%)$ \\
\hline Yes, full-time & $65(6.4 \%)$ \\
\hline Type of school, $N(\%)$ & \\
\hline Residential & $44(35.2 \%)$ \\
\hline Commuter & $51(40.8 \%)$ \\
\hline Online-only & $30(24.0 \%)$ \\
\hline Prior to COVID-19 employment status, & \\
\hline No & $180(17.7 \%)$ \\
\hline Yes, part-time & $185(18.2 \%)$ \\
\hline Yes, full-time & $650(64.0 \%)$ \\
\hline Current employment status, $N(\%)$ & \\
\hline No & $286(28.2 \%)$ \\
\hline Yes, part-time & $198(19.5 \%)$ \\
\hline Yes, full-time & $531(52.3 \%)$ \\
\hline Finances sufficient to meet needs, $N(\%)$ & \\
\hline Not at all & $74(7.3 \%)$ \\
\hline A little & $209(20.6 \%)$ \\
\hline Moderately & $219(21.6 \%)$ \\
\hline Mostly & $276(27.2 \%)$ \\
\hline Completely & $237(23.3 \%)$ \\
\hline
\end{tabular}


Table 2 Prevalence and Stressfulness of COVID-19-Related Stressors

\begin{tabular}{|c|c|c|c|c|}
\hline & $\begin{array}{l}N \text { endorsed } \\
(\%)\end{array}$ & $\begin{array}{l}\text { Group differences in } \\
\text { stressor prevalence }\end{array}$ & $\begin{array}{l}\text { Mean stress } \\
\text { appraisal }(S D)\end{array}$ & $\begin{array}{l}\text { Group differences } \\
\text { in stress appraisal }\end{array}$ \\
\hline \multicolumn{5}{|l|}{ Infection-related } \\
\hline 1. Risk of becoming infected & $576(56.8 \%)$ & $\mathrm{e} 1(r=-.09)$ & $3.06(1.03)$ & $\begin{array}{l}\text { a1 }(d=-0.26) \\
\mathrm{b}(d=0.20) \\
\mathrm{e} 1(r=-.09)\end{array}$ \\
\hline 2. Self-monitoring of symptoms & $440(43.4 \%)$ & $\begin{array}{l}\text { c1 }(h=0.36) \\
\text { e1 }(r=-.07)\end{array}$ & $2.43(1.13)$ & $\begin{array}{l}\mathrm{b}(d=0.36) \\
\mathrm{d} 1(r=-.18) \\
\text { e1 }(r=-.18) \\
\mathrm{f}(d=0.28)\end{array}$ \\
\hline 3. Risk of loved ones becoming infected & $622(61.3 \%)$ & al $(h=-0.14)$ & $3.65(1.01)$ & $\begin{array}{l}\text { a } 1(d=-0.23) \\
\text { b }(d=0.21) \\
\text { e1 }(r=-.21)\end{array}$ \\
\hline 4. Risk of unintentionally infecting other people & $246(24.3 \%)$ & $\begin{array}{l}\mathrm{d} 1(r=-.12) \\
\mathrm{e} 1(r=-.07)\end{array}$ & $3.26(1.08)$ & $\mathrm{e} 1(r=-.16)$ \\
\hline $\begin{array}{l}\text { 5. Read or heard others talk about the severity and } \\
\text { contagiousness of COVID-19 }\end{array}$ & $980(96.6 \%)$ & $\mathrm{d} 2(r=.07)$ & $3.07(1.08)$ & $\begin{array}{l}\text { a1 }(d=-0.23) \\
\text { d1 }(r=-.09) \\
\text { e1 }(r=-.15)\end{array}$ \\
\hline $\begin{array}{l}\text { 6. Stigma, shame, discrimination, or social exile related to } \\
\text { quarantine or working in a high-risk area } \\
\text { (e.g., others shunning you because you work in healthcare)? }\end{array}$ & $66(6.5 \%)$ & $\begin{array}{l}\mathrm{b}(h=0.23) \\
\mathrm{d} 1(r=-.12)\end{array}$ & $3.53(0.96)$ & $\mathrm{b}(d=0.53)$ \\
\hline $\begin{array}{l}\text { 7. Stigma, shame, or discrimination related to being in a } \\
\text { certain age group (e.g., negative statements about Millennials } \\
\text { or Generation } Z \text { ) }\end{array}$ & $158(15.6 \%)$ & $\begin{array}{l}\text { c1 }(h=0.20) \\
\text { d1 }(r=-.22) \\
\text { e1 }(r=-.10)\end{array}$ & $2.57(1.13)$ & $\mathrm{b}(d=0.40)$ \\
\hline $\begin{array}{l}8 \text {. Uncertainty about how long quarantine and/or social } \\
\text { distancing requirements will last? }\end{array}$ & $896(88.3 \%)$ & a1 $(h=-0.13)$ & $3.26(1.08)$ & $\begin{array}{l}\text { a1 }(d=-0.25) \\
\text { d1 }(r=-.20) \\
\text { e1 }(r=-.17)\end{array}$ \\
\hline \multicolumn{5}{|l|}{ Activity-related } \\
\hline $\begin{array}{l}\text { 9. Changes to daily personal care routines } \\
\text { (e.g., cooking, cleaning, exercise/relaxation, hobbies) }\end{array}$ & $811(80.1 \%)$ & $\mathrm{d} 1(r=-.08)$ & $2.76(1.12)$ & $\begin{array}{l}\text { a1 }(d=-0.15) \\
\text { b }(d=0.17) \\
\text { d1 }(r=-.20) \\
\text { e1 }(r=-.22)\end{array}$ \\
\hline $\begin{array}{l}\text { 10. Changes to daily work routines } \\
\text { (e.g., unable to earn money, attend full- or part-time work } \\
\text { schedule) }\end{array}$ & $608(60.3 \%)$ & $\begin{array}{l}\mathrm{d} 1(r=-.18) \\
\mathrm{e} 1(r=-.15)\end{array}$ & $3.46(1.16)$ & $\begin{array}{l}\text { a1 }(d=-0.32) \\
\text { d1 }(r=-.17) \\
\text { e1 }(r=-.39)\end{array}$ \\
\hline $\begin{array}{l}\text { 11. Changes to daily education routines } \\
\text { (e.g., online instruction) }\end{array}$ & $233(23.0 \%)$ & $\begin{array}{l}\text { a1 }(h=-0.25) \\
\text { b }(h=0.43) \\
\text { d1 }(r=-.20)\end{array}$ & $3.16(1.13)$ & $\mathrm{d} 1(r=-.13)$ \\
\hline $\begin{array}{l}\text { 12. Changes to social routines } \\
\text { (e.g., spending free time with friends/loved ones) }\end{array}$ & $849(83.7 \%)$ & - & $2.91(1.12)$ & $\begin{array}{l}\text { a1 }(d=-0.22) \\
\text { d1 }(r=-.10) \\
\text { e1 }(r=-.13)\end{array}$ \\
\hline $\begin{array}{l}\text { 13. Changed responsibilities to care for dependents } \\
\text { (e.g., childcare, eldercare) }\end{array}$ & $246(24.3 \%)$ & $\begin{array}{l}\mathrm{b}(h=1.02) \\
\mathrm{c} 2(h=-0.26)\end{array}$ & $2.10(1.17)$ & $\begin{array}{l}\mathrm{d} 1(r=-.23) \\
\mathrm{e} 1(r=-.18)\end{array}$ \\
\hline $\begin{array}{l}\text { 14. Cancelation of planned or scheduled celebrations, } \\
\text { entertainment, vacations, or trips } \\
\text { (e.g., graduations, birthdays, concerts) }\end{array}$ & $696(68.7 \%)$ & $\begin{array}{l}\text { a1 }(h=-0.27) \\
\text { b }(h=0.25) \\
\text { d1 }(r=-.07)\end{array}$ & $2.95(1.15)$ & $\mathrm{d} 1(r=-.11)$ \\
\hline $\begin{array}{l}\text { 15. Cancelation of meaningful personal or religious rituals } \\
\text { (e.g., funerals, religious services) }\end{array}$ & $266(26.3 \%)$ & $\begin{array}{l}\mathrm{b}(h=0.32) \\
\mathrm{c} 2(h=-0.42) \\
\mathrm{d} 2(r=.08) \\
\mathrm{f}(h=0.16)\end{array}$ & $2.94(1.19)$ & $\begin{array}{l}\text { a1 }(d=-0.34) \\
\text { e1 }(r=-.18)\end{array}$ \\
\hline $\begin{array}{l}\text { 16. Inability to travel } \\
\text { (e.g., cancelation of vacations, weekend trips) }\end{array}$ & $565(55.8 \%)$ & $\mathrm{e} 2(r=.07)$ & $2.90(1.12)$ & $\mathrm{d} 1(r=-.12)$ \\
\hline $\begin{array}{l}\text { 17. Increased contact with close others or loved ones } \\
\text { (e.g., increased conflict, co-worrying) }\end{array}$ & $438(43.3 \%)$ & $\begin{array}{l}\mathrm{b}(h=0.20) \\
\mathrm{c} 1(h=0.24)\end{array}$ & $2.60(1.17)$ & $\begin{array}{l}\text { a1 }(d=-0.28) \\
\text { c1 }(d=0.27) \\
\text { d1 }(r=-.19) \\
\text { e1 }(r=-.22)\end{array}$ \\
\hline $\begin{array}{l}\text { 18. Pressure to "make the most of" COVID-19 or "find a } \\
\text { silver lining" while quarantining (e.g., social media fitness } \\
\text { challenges, encouragement to increase productivity) }\end{array}$ & $502(49.7 \%)$ & $\begin{array}{l}\text { a1 }(h=-0.24) \\
\text { b }(h=0.18) \\
\text { c1 }(h=0.20) \\
\text { d1 }(r=-.11)\end{array}$ & $2.41(1.20)$ & $\begin{array}{l}\text { a1 }(d=-0.28) \\
\text { d1 }(r=-.19) \\
\text { e1 }(r=-.18)\end{array}$ \\
\hline \multicolumn{5}{|l|}{ Financial/resource-related } \\
\hline $\begin{array}{l}\text { 19. Loss of current job security or income } \\
\text { (e.g., inability to earn money)? }\end{array}$ & $339(33.6 \%)$ & $\begin{array}{l}\text { a1 }(h=-0.11) \\
\text { c1 }(h=0.20) \\
\text { d1 }(r=-.11) \\
\text { e1 }(r=-.37)\end{array}$ & $4.09(0.96)$ & $\begin{array}{l}\mathrm{d} 1(r=-.16) \\
\mathrm{e} 1(r=-.32)\end{array}$ \\
\hline $\begin{array}{l}\text { 20. Loss of current job training opportunities or education } \\
\text { benchmarks (e.g., certification, apprenticeship, internship or } \\
\text { degree completion) }\end{array}$ & $121(12.0 \%)$ & $\begin{array}{l}\mathrm{d} 1(r=-.22) \\
\mathrm{e} 1(r=-.13)\end{array}$ & 3.52 (1.18) & $\begin{array}{l}\text { a1 }(d=-0.42) \\
\text { e1 }(r=-.33)\end{array}$ \\
\hline $\begin{array}{l}\text { 21. Potential changes to the national or global economy } \\
\text { (e.g., future job prospects, loss of investments) }\end{array}$ & $643(63.6 \%)$ & - & $3.45(1.05)$ & $\begin{array}{l}\text { a1 }(d=-0.20) \\
\text { d1 }(r=-.12) \\
\text { e1 }(r=-.19)\end{array}$ \\
\hline $\begin{array}{l}\text { 22. Difficulty accessing important resources for daily life } \\
\text { (e.g., healthcare, food, clothes, water, housing, medical } \\
\text { supplies or prescriptions) }\end{array}$ & $501(49.5 \%)$ & $\begin{array}{l}\text { a1 }(h=-0.21) \\
\text { e1 }(r=-.23)\end{array}$ & $3.36(1.04)$ & $\begin{array}{l}\text { a1 }(d=-0.28) \\
\text { d1 }(r=-.18) \\
\text { e1 }(r=-.20)\end{array}$ \\
\hline $\begin{array}{l}\text { 23. Inadequate access to reliable information about COVID- } \\
19 \text { (including your personal risk of illness) }\end{array}$ & $176(17.4 \%)$ & $\begin{array}{l}\mathrm{d} 1(r=-.10) \\
\mathrm{e} 1(r=-.15)\end{array}$ & $3.50(1.09)$ & $\mathrm{e} 1(r=-.23)$ \\
\hline
\end{tabular}


$a 1$, reported more by women vs. men; a2, reported more by men vs. women; $b$, reported more by caregivers vs. non-caregivers; $c 1$, reported more by non-heterosexual participants; 2 , reported more by heterosexual participants; $d 1$, negatively associated with older age; d2, positively associated with older age; e1, negatively associated with having needs met; e2, positively associated with having needs met; f, reported more often by non-White participants (all two-tailed p's <.05). Two-tailed independent samples t tests were used for categorical group comparisons; bivariate correlations were used for continuous predictors (age and financial needs met) to retain maximal variance. Average stress appraisals apply only for participants who endorsed experiencing each item; participants who did not endorse a given item did not report on stress appraisals. Standardized effect sizes differ by statistical test used $(h=$ Cohen's $h(0.20=$ small, $0.50=$ medium, $0.80=$ large $) ; d=$ Cohen's $d(0.20=$ small, $0.50=$ medium, $0.80=$ large $) ; r=$ bivariate correlation $(0.1=$ small, $0.3=$ medium, $0.5=$ large $))$

reading/hearing about the severity and contagiousness of COVID-19 (96.6\%), uncertainty about length of quarantine and social distancing requirements $(88.3 \%)$, and changes to social $(83.7 \%)$ and daily personal care $(80.1 \%)$ routines. Individuals who reported financial strain appear to be at a particular risk for stressor exposure during the COVID-19 pandemic (particularly infectionrelated risk as well as lack of job security and lack of resources). Age, gender, and caregiver status were also important predictors of stress exposure across several domains (Cohen's $h$ for all significant effects ranging from 0.11 to $-0.43 ; r$ 's ranging from -0.07 to -0.37 ). Of note, participants who cared for dependents were much more likely to experience past-week changes in caregiving demands related to COVID-19 $(h=1.02,95 \%$ CI $[0.90$, 1.14 ], $p<.001)$. Of the stressors experienced, the most stressful was loss of job security or income $(M=4.09, S D$ $=0.96)$, risk of a loved one's illness $(M=3.65, S D=$ 1.01), stigma related to being high risk $(M=3.53, S D=$ $0.96)$, loss of job/education $(M=3.52, S D=1.18)$, and lack of access to information $(M=3.50, S D=1.09)$.

Similar to trends in stress exposure, women appraised events as significantly more stressful than did men across multiple domains, including infection-related risk, change to daily activities, and resource insecurity ( $d$ 's ranging from -0.15 to -0.47$)$. Caregivers reported significantly greater stress appraisals primarily in relation to infectionrelated risk as well as moderately greater stress regarding perceived stigma of being quarantined or working in a high risk context $(d=0.53,95 \%$ CI [0.01, 1.05], $p=.04$; all other significant $d$ 's for caregivers ranged from 0.17 to $0.40)$. Non-specific to domain, younger participants and those with fewer resources also reported a high number of stressors as more stressful than did older, better-resourced participants (significant $r$ 's range from -.09 to -.23 for age; from -.15 to -.39 for financial resources), sexual minority participants were more stressed by rumination and co-worry with others $(d=0.27,95 \%$ CI $[0.01$, $0.54], p=.04)$, and non-White participants experienced more stress related to symptom self-monitoring $(d=0.28$, $95 \%$ CI $[0.02,0.54], p=.03$ ).

\section{COMMON COPING STRATEGIES}

The most common coping strategies were distraction, active coping, and seeking emotional social support (Table 3). Female gender was associated with greater use of multiple emotion-focused strategies such as distraction, emotional and religious support, and less use of humor ( $d$ 's range from 0.22 to -0.31 ). Caregivers similarly reported greater emotion and religious support coping, but also exhibited significantly greater use of active strategies and instrumental social support seeking ( $d$ 's range from $=0.16$ to 0.21 ). Younger participants, sexual minorities, and those with greater financial instability generally reported greater use of less productive strategies, including substance use, behavioral disengagement, and humor, all with very small to medium effect sizes (significant $d$ 's

Table 3 Coping with COVID-Related Stress

\begin{tabular}{|c|c|c|c|c|}
\hline & Mean $(S D)$ & Range & Cronbach's alpha & Demographic predictors \\
\hline \multicolumn{5}{|l|}{ COPE subscales } \\
\hline 1. Substance use (4 items) & $1.45(0.78)$ & $1-4$ & 0.96 & $\mathrm{c} 1(d=0.24), \mathrm{d} 1(r=-.20), \mathrm{e} 1(r=-.12)$ \\
\hline 2. Active (2 items) & $2.43(0.83)$ & $1-4$ & 0.77 & $\mathrm{~b}(d=0.18), \mathrm{c} 2(d=-0.19)$ \\
\hline 3. Distraction (2 items) & $2.70(0.80)$ & $1-4$ & 0.48 & a1 $(d=-0.31), \mathrm{d} 1(r=-.10)$ \\
\hline 4. Behavioral disengagement ( 2 items) & $1.40(0.66)$ & $1-4$ & 0.74 & $\mathrm{c} 1(d=0.24), \mathrm{d} 1(r=-.24), \mathrm{e} 1(r=-.15)$ \\
\hline 5. Humor (2 items) & $1.86(0.89)$ & $1-4$ & 0.88 & $\mathrm{a} 2(d=0.22), \mathrm{c} 1(d=0.24), \mathrm{d} 1(r=-.18)$ \\
\hline 6. Seeking instrumental social support (2 items) & $1.91(0.83)$ & $1-4$ & 0.83 & $\mathrm{~b}(d=0.19), \mathrm{d} 1(r=-.15)$ \\
\hline 7. Seeking emotional social support (2 items) & $2.23(0.89)$ & $1-4$ & 0.84 & a1 $(d=-0.24), \mathrm{b}(d=0.16)$ \\
\hline 8. Religious support (2 items) & $1.78(1.00)$ & $1-4$ & 0.90 & $\begin{array}{l}\mathrm{a} 1(d=-0.30), \mathrm{b}(d=0.21), \\
\mathrm{c} 2(d=-0.31), \mathrm{d} 2(r=.13), \mathrm{f}(d=0.27)\end{array}$ \\
\hline
\end{tabular}

$a 1$, reported more by women vs. men; a2, reported more by men vs. women; $b$, reported more by caregivers vs. non-caregivers; $c 1$, reported more by non-heterosexual participants; $c 2$, reported more by heterosexual participants; $d 1$, negatively associated with older age; d2, positively associated with older age; e1, negatively associated with having needs met; e2, positively associated with having needs met; $f$, reported more often by non-White participants (all two-tailed p's <.05). Two-tailed independent samples t tests were used for categorical group comparisons; bivariate correlations were used for continuous predictors (age and financial needs met). Mean response values were calculated for each subscale to facilitate comparison and interpretability. Standardized effect sizes differ by statistical test used ( $d=$ Cohen's $d(0.20=$ small, $0.50=$ medium, $0.80=$ large); $r=$ bivariate correlation $(0.1=$ small, $0.3=$ medium, $0.5=$ large $))$ 
Table 4 Adherence with CDC Recommendations

\section{=}

1. Avoid in person social gatherings

2. Avoid eating or gathering at bars, restaurants and food courts - use drive thru, pickup, or delivery options instead

3. Avoid discretionary travel, shopping trips, and social visits

4. Avoid visits to nursing homes, retirement homes, and long-term care facilities

5. Put $6 \mathrm{ft}$ of physical distance between yourself and other people

6. Avoid physical touching when greeting other people (i.e., handshakes, hugs)

7. Avoid touching your eyes, nose and mouth with unwashed hands

8. Avoid close contact with people who are sick

9. Cover your mouth and nose with a tissue when you cough or sneeze

(or use the inside of your elbow); throw used tissues in the trash

10. Clean and disinfect frequently touched surfaces daily

11. Wash your hands often, with soap and water for at least $20 \mathrm{~s}$, or if soap and water are not readily available, use a hand sanitizer that contains at least $60 \%$ alcohol. Cover all surfaces of your hands and rub them together until they feel dry

12. Have a plan for taking time off from work or school, in case you are told to stay home for 14 days of self-monitoring or if you get sick with COVID-19 $N$ reporting yes $(\%)$

Have you felt unwell/sick or had a fever in the last 7 days or tested positive for COVID-19?

1. Wear a facemask

2. Stay home, except to get medical care

\begin{tabular}{|c|c|c|}
\hline $\begin{array}{l}\text { Mean (SD) \% } \\
\text { adherent } \\
91.99(18.50)\end{array}$ & $\begin{array}{l}\text { Median \% } \\
\text { adherent } \\
100\end{array}$ & $\begin{array}{l}\text { Demographic } \\
\text { predictors } \\
\text { a1 }(d=-0.16), \\
\text { d2 }(r=.10), \\
\text { e2 }(r=.09)\end{array}$ \\
\hline $95.10(15.57)$ & 100 & $\begin{array}{l}\text { a1 }(d=-0.13), \\
\text { d2 }(r=.15), \\
\text { e2 }(r=.08)\end{array}$ \\
\hline 89.74 (19.55) & 100 & $\begin{array}{l}\text { a1 }(d=-0.27), \\
\text { d2 }(r=.08), \\
\text { e2 }(r=.09)\end{array}$ \\
\hline 94.38 (19.17) & 100 & $\begin{array}{l}\mathrm{a} 1(d=-0.16), \\
\mathrm{d} 2(r=.09)\end{array}$ \\
\hline 87.42 (19.84) & 97 & $\begin{array}{l}\text { a1 }(d=-0.18), \\
\text { d2 }(\mathrm{r}=.17), \\
\text { e2 }(r=.07)\end{array}$ \\
\hline 93.06 (17.33) & 100 & $\begin{array}{l}\mathrm{d} 2(r=.15) \\
\mathrm{e} 2(r=.10)\end{array}$ \\
\hline 77.87 (25.30) & 85 & $\mathrm{~d} 2(r=.08)$ \\
\hline $94.86(15.53)$ & 100 & $\begin{array}{l}\mathrm{d} 2(r=.12) \\
\mathrm{e} 2(r=.06)\end{array}$ \\
\hline 91.07 (19.34) & 100 & $\begin{array}{l}\text { a1 }(d=-0.24), \\
\text { d2 }(r=.11)\end{array}$ \\
\hline 74.68 (29.88) & 87 & $\begin{array}{l}\text { a1 }(d=-0.23), \\
\text { b }(d=0.28), \\
\text { c2 }(d=-0.39), \\
\text { d2 }(r=.15)\end{array}$ \\
\hline $88.27(20.25)$ & 99 & $\begin{array}{l}\mathrm{a} 1(d=-0.16), \\
\mathrm{b}(d=0.16), \\
\mathrm{d} 2(r=.09)\end{array}$ \\
\hline $72.74(35.85)$ & 97 & $\mathrm{e} 2(r=.12)$ \\
\hline
\end{tabular}

$62(6.1)$

Average (SD) \% compliance $50.00(40.49)$

$84.87(26.05)$

\section{Median \% compliance 51 \\ 97}

\section{Demographic predictors}

al, reported more by women vs. men; a2, reported more by men vs. women; $b$, reported more by caregivers vs. non-caregivers; cl, reported more by non-heterosexual participants; c2, reported more by heterosexual participants; $d 1$, negatively associated with older age; d2, positively associated with older age; e1, negatively associated with having needs met; e2, positively associated with having needs met; $f$, reported more often by non-White participants (all two-tailed p's <.05). Two-tailed independent samples t tests were used for categorical group comparisons; bivariate correlations were used for continuous predictors (age and financial needs met). Standardized effect sizes differ by statistical test used $(d=C o h e n ' s d(0.20=s m a l l, 0.50$ $=$ medium, $0.80=$ large $) ; r=$ bivariate correlation $(0.1=$ small, $0.3=$ medium, $0.5=$ large $))$

range from -0.19 to $-0.31 ; r$ 's range from -0.10 to $0.24)$.

\section{ADHERENCE TO CDC GUIDELINES: SOCIAL DISTANCING AND HEALTH BEHAVIOR/HYGIENE}

Adherence to CDC guidelines was generally fairly high, but uneven (Table 4). Respondents reported a high average level of compliance with several aspects of social distancing, including avoiding eating out or gathering at bars/restaurants (95.10\%) and avoiding visiting nursing homes $(94.38 \%)$, although these rates may be a result of municipal restrictions to essential businesses. Lower but still robust adherence was noted for social distancing (e.g., discretionary travel $(89.74 \%)$, keeping 6 - $\mathrm{ft}$ distance $(87.42 \%)$ ) and hygiene (e.g., touching face (77.87\%), cleaning/disinfecting (74.68\%)). Across adherence items, older age, female gender, and financial security were the most consistent predictors of adherence to CDC guidelines, with other significant predictors emerging at the item level (e.g., heterosexual status predicting more daily cleaning of surfaces) albeit with very small to small effect sizes (significant $d$ 's range from -0.13 to -0.39 ; $r$ 's range from .06 to .17 ).

\section{CONCLUSION}

This study is among the first to present reports of stress and coping responses to COVID-19 in the USA and adherence to CDC guidelines. Similar to prior studies of COVID- $19^{3}$ and $\mathrm{SARS}^{19}$ in China, people in the USA reported experiencing many stressors in all aspects of their lives: in addition to nearly universal reports of stress over the spread of the disease, uncertainty and changes in routines were highly prevalent. Although reported less frequently, financial concerns (worry over job security and lack of resources) stand out as among the most stressful. 
Distraction, the most commonly reported coping strategy, is often used following major traumatic events (e.g., 9/11) (3) $^{23}$ and can be helpful in situations of low control. ${ }^{24}$ Perhaps relying on distraction reflects limitations in what people can do given social distancing practices (including shelter in place and nonessential business closures in many communities). Frequent use of active coping and emotional support-seeking strategies are encouraging, even if much of it is likely telemediated. Overall, reported coping strategies indicate that people are employing many adaptive coping strategies to manage their stress.

Rate of adherence with CDC guidelines overall seems quite high compared with general population adherence to other types of public health guidelines (e.g., ${ }^{25}$ ), but an expected level of adherence in acute situations such as the current pandemic has no precedent upon which to draw. Additionally, some of the reported behaviors indicate a potentially worrisome lack of adherence to critical CDC guidelines - particularly for men and younger adults.

Limitations of the current study must be acknowledged. Generalizability is limited by the composition of our sample of MTurk workers. While men and women are relatively equally represented among MTurk workers, are, on average, slightly younger than the US population as a whole $(88 \%$ of workers are under 50 compared with $66 \%$ of employed adults) and better educated (51\% of workers have college degrees compared with $36 \%$ of US adults over 18); about 3/4 of MTurk workers are White and non-Hispanic compared with $66 \%$ of the US population. ${ }^{13,14,26}$ While the sample cannot be characterized as nationally representative, these results offer valuable insight into early indicators of risk and vulnerability to COVID-19-related stressors. The measures used in this study were also very brief and therefore excluded many likely important factors. Although adequately statistically powered, results should be interpreted with caution given some of the small effect sizes; these results warrant replication with longer instruments and multivariate modeling.

Our findings may inform future policy and interventions particularly given that they suggest heightened vulnerability in key subgroups. Specifically, we draw attention to the need for future work to identify and support caregivers, younger adults, and other minority and disadvantaged groups (e.g., sexual minority and lower-resourced individuals), as these segments of our sample indicated higher stress, lower compliance with prevention behaviors, and/or less adaptive coping strategies. Based on responses to previous disasters, expectations are of substantial long-term mental health problems. ${ }^{27}$ These data, collected as the COVID-19 pandemic was gaining steam nationally, underscore the need for more resources to help individuals create healthy and safe coping responses as the virus continues to spread as well as the need to prepare for increased demands for mental health services long after the pandemic is brought under control.
Finally, we note that reading/hearing about the severity and contagiousness of COVID-19 was by far the most commonly experienced stressor in our sample, underscoring that COVID-19 is spreading in the context of a social-media environment that rapidly connects the global population to ongoing messages about the virus. The same environment, however, may create the conditions for effective online communication and dissemination of resources to help those who are particularly vulnerable to cope more adaptively in the coming months. ${ }^{28}$

Corresponding Author: Crystal L. Park, Ph.D.; Department of Psychological Sciences, University of Connecticut, Mansfield, CT, United States (e-mail: crystal.park@uconn.edu).

Funding Information We received support for this project from NIH (NIAAA 1R34AA027455) and a seed grant from UCONN InCHIP.

\section{Compliance with Ethical Standards:}

All study materials were approved by the University of Connecticut IRB (X20-0057) as an exempt protocol.

Conflict of Interest: The authors declare that they do not have a conflict of interest.

\section{REFERENCES}

1. Coronavirus (COVID-19): Daily life and coping. Centers for Disease Control, 2020a. (https://www.cdc.gov/coronavirus/2019-ncov/dailylife-coping/index.html.)

2. Brooks SK, Webster RK, Smith LE, et al. The psychological impact of quarantine and how to reduce it: rapid review of the evidence. Lancet 2020;395:912-20.

3. Giu J, Shen B, Zhao M, Wang Z, Xie B, \& Xu Y. A nationwide survey of psychological distress among Chinese people in the COVID-19 epidemic: Implications and policy recommendations. Gen Psychiatry 2020;33:e100213.

4. Zhang Y, Ma ZF. Impact of the COVID-19 Pandemic on Mental Health and Quality of Life among Local Residents in Liaoning Province, China: A Cross-Sectional Study. Int $J$ of Env Rsch and Pub Hlth. 2020 Jan; 17(7):2381.

5. Wang C. Pan R, Wan X. et al. Immediate psychological responses and associated factors during the initial stage of the 2019 coronavirus disease (COVID-19) epidemic among the general population in china. In J o f Env Res Pub Health 2020;17:1729.

6. Sun J, Harris K, \& Vazire S. Is well-being associated with the quantity and quality of social interactions? J of Pers and Soc Psych 2019; Advance online publication. https://doi.org/10.1037/pspp0000272

7. Coronavirus (COVID 19): How to protect yourself and others. Centers for Disease Control, 2020b. (https://www.cdc.gov/coronavirus/2019-ncov/ prevent-getting-sick/prevention.html.)

8. Baumeister RF, \& Leary MR. The need to belong: Desire for interpersonal attachments as a fundamental human motivation. Psych Bull 1995;117:497-529.

9. Aldwin, CM (2007). Stress, coping, and development. New York, NY: Guilford.

10. Finkelstein-Fox L, Park CL. Control-coping goodness-of-fit and chronic illness: a systematic review of the literature. Health Psych Rev. 2019 Apr 3;13(2):137-62.

11. Stoycheff E. Please participate in Part 2: Maximizing response rates in longitudinal MTurk designs. Meth Innov 2016;9:1-5.

12. Bartneck C, Deunset A, Moltchanova E, \& Zawieska K. Comparing the similarity of responses received from studies in Amazon's Mechanical Turk to studies conducted online and with direct recruitment. PLoS ONE 2015;10:1-23. 
13. Huff C, \& Tingley D. "Who are these people?" Evaluating the demographic characteristics and political preferences of MTurk survey respondents. Res \& Politics 2015;2:1-12.

14. Sheehan KB, \& Pittman M. Amazon's mechanical Turk for academics: The HIT handbook for social science research. Irvine, CA: Melvin \& Leigh, 2016.

15. Berinsky AJ, Huber GA, \& Lenz GS. Evaluating online labor markets for experimental research: Amazon.com's Mechanical Turk. Pol Analysis 2012;20:351-68.

16. Kees J, Berry C, Burton $\mathbf{S}$, \& Sheehan $\mathbf{K}$. An analysis of data quality: Professional panels, student subject pools, and Amazon's mechanical Turk. J of Advertising 2017;46:141-55.

17. Mortensen K, Hughes TL. Comparing Amazon's Mechanical Turk platform to conventional data collection methods in the health and medical research literature. J of Gen Int Med. 2018 Apr 1;33(4):533-8.

18. U. S. Census Regions and Divisions of the United States [Internet], United States Census Bureau, April 11 2020. (https://www2.census.gov/geo/ pdfs/maps-data/maps/reference/us_regdiv.pdf.)

19. Main A, Zhou Q, Ma Y, Luecken LJ, \& Liu X. Relations of SARS-related stressors and coping to Chinese college students' psychological adjustment during the 2003 Beijing SARS epidemic. J of Couns Psych 2011;58:410-23.

20. Carver CS. You want to measure coping but your protocol's too long: Consider the Brief COPE. Int J of Beh Med 1997;4:92-100.

21. DeSimone JA, Harms PD, \& DeSimone AJ. Best practice recommen dations for data screening. $\mathrm{J}$ of Org Beh 2015;36:171-81.

22. Sheehan KB. Crowdsourcing research: Data collection with Amazon's Mechanical Turk. Comm Monographs 2018;85:140-56.
23. Butler LD, Blasey CM, Garlan RW, et al. Posttraumatic growth following the terrorist attacks of September 11, 2001: Cognitive, coping, and trauma symptom predictors in an internet convenience sample. Traumatology. 2005 Dec;11(4):247-67.

24. Janson J, Rohleder $\mathbf{N}$. Distraction coping predicts better cortisol recovery after acute psychosocial stress. Biol Psych. 2017 Sep $1 ; 128: 117-24$

25. Powell-Wiley TM, Miller PE, Agyemang P, Agurs-Collins T \& Reedy J. Perceived and objective diet quality in US adults: a cross-sectional analysis of the National Health and Nutrition Examination Survey (NHANES). Pub Health Nutr 2014;17:2641-49.

26. Hitlin, H. Research in the crowdsourcing age, a case study: How scholars, companies and workers are using Mechanical Turk, a 'gig economy' platform, for tasks computers can't handle. Pew Research Center, July 2016. (http://www.pewinternet.org/2016/07/11/researchin-the-crowdsourcing-age-a-case-study/).

27. Galea S, Merchant RM, Lurie N. The Mental Health Consequences of COVID-19 and Physical Distancing: The Need for Prevention and Early Intervention. JAMA Internal Medicine.2020 April $10 \mathrm{https}$ //doi.org/10. 1001/jamainternmed.2020.1562

28. Merchant RM, Lurie N. Social media and emergency preparedness in response to novel coronavirus. JAMA. 2020 Mar 23. https://doi.org/10. $1001 /$ jama.2020.4469

Publisher's Note: Springer Nature remains neutral with regard to jurisdictional claims in published maps and institutional affiliations. 